\title{
Standounkt
}

Klimaschutzpolitik und Automobilität

\section{Wie die Bundesregierung den Klimaschutz und sparsame Neuwagen ausbremst}

\author{
Die Bundesregierung hat erneut die Verschärfung von Abgas- \\ normen verzögert, obwohl diese von der EU bereits vereinbart \\ wurden. Damit macht sich die Bundesregierung zum Hand- \\ langer der Autolobby. Von Michael Müller-Görnert
}

$E^{n}$ nde Juni hatte sich die Europäische Union (EU) auf einen Kompromiss geeinigt, die seit 2012 geltenden Grenzwerte für den Ausstoß von Kohlenstoffdioxid $\left(\mathrm{CO}_{2}\right)$ von neuen Pkw für die Zeit ab 2020 fortzuschreiben. Bis 2015 dürfen Neuwagen im Schnitt nicht mehr als 130 Gramm $\mathrm{CO}_{2}$ pro Kilometer emittieren. Ab 2020 soll dieser Wert auf 95 Gramm sinken. Das entspricht einem Durchschnittsverbrauch von rund vier Litern.

Schon heute lässt sich mit sparsamen Fahrzeugen aus allen Fahrzeugsegmenten eine Flotte zusammenstellen, die bereits den künftigen Wert von 95 Gramm pro Kilometer einhält. Der ökologische Verkehrsclub (VCD) und weitere Umwelt- und Verbraucherverbände hatten daher im Vorfeld einen ambitionierteren Grenzwert von 80 Gramm gefordert.

\section{Kompromiss blockiert}

Die Bundesregierung hat den ausgehandelten Kompromiss allerdings platzen lassen und Bundeskanzlerin Angela Merkel höchstpersönlich hat dafür gesorgt, die formale Bestätigung des Textes durch die EU-Botschafter von der Tagesordnung zu nehmen. Dabei wurden einige EU-Länder unter Druck gesetzt oder es wurde ihnen Entgegenkommen bei anderen Politikfeldern signalisiert. Während auf europäischer Ebene der Einigungswille da ist, blockiert Deutschland bisher mit aller Macht eine Abstimmung. Vielmehr wird versucht, ein Aufweichen der Regelung zugunsten der deutschen
Premiumhersteller BMW und Daimler zu erreichen.

Der ausgehandelte Kompromiss kommt der Autoindustrie bereits weit entgegen, indem Autohersteller jedes im Jahr 2020 verkaufte Elektro-Auto doppelt auf ihre $\mathrm{CO}_{2}$-Bilanz anrechnen können. Diese Mehrfachanrechnung sollte bis 2023 nach und nach auslaufen. Da reine batterieelektrische Fahrzeuge ohne zusätzlichen Verbrennungsmotor als Nullemissionsautos gelten, gleicht so jedes Elektroauto ein Fahrzeug mit hohem Verbrauch aus. Dies reichte BMW und Daimler offenbar nicht. Besonders schamlos ist der jüngste deutsche Vorschlag, den Grenzwert bis 2024 schrittweise einzuführen. Dies bedeutet de facto eine Verschiebung um vier Jahre.

\section{Handlanger der Autoindustrie}

Die einst gelobte Klimakanzlerin macht sich durch ihr Eingreifen zum Handlanger der deutschen Autoindustrie - gegen die Interessen des Klimaschutzes und der Verbraucherinnen und Verbraucher. Das ist ein Skandal! Nicht nur Umwelt- und Verbraucherverbände kritisierten das dreiste Vorgehen von Merkel und Co, sondern auch zahlreiche EU-Diplomaten und der Vorsitzende des Umweltausschusses im Europaparlament, Matthias Groote. Nun droht die Gefahr, dass die Grenzwerte auf den Sankt Nimmerleinstag verschoben werden, denn angesichts der Europawahlen im kommenden Jahr müssten dann die neu gewählten EU-Parlamentarier sich mit dem Thema beschäftigen.

Ambitionierte $\mathrm{CO}_{2}$-Grenzwerte sind eines der effektivsten Instrumente, um den Ausstoß von Treibhausgasen im Verkehr zu verringern und gleichzeitig den Spritverbrauch zu senken. Der Volkswagenkonzern, Europas größter Autohersteller, kann mit dem auf EU-Ebene verhandelten Kompromiss von 95 Gramm $\mathrm{CO}_{2}$ pro Kilometer ohne Wenn und Aber leben; auch die Zulieferindustrie hat sich dazu bekannt, denn sie profitiert genau wie die Verbraucher von $\mathrm{CO}_{2}$-Grenzwerten. Autofahrerinnen und Autofahrer sparen bei einem Grenzwert von 95 Gramm jährlich zwischen 500 und 900 Euro.

\section{Verkehr als Sorgenkind der Klimaschutzpolitik}

Der Verkehr gehört nach wie vor zu den Sorgenkindern der Klimaschutzpolitik. Während in allen anderen Sektoren die Treibhausgasemissionen in den letzten Jahren teilweise deutlich verringert wurden, sind sie im Verkehrsbereich gestiegen. Die zunehmenden Extremwetterereignisse - wie die zweite Flutkatastrophe in Deutschland innerhalb von elf Jahren - zeigen jedoch, wie wichtig es ist, den Treibhausgasausstoß drastisch zu verringern.

Wenn wir nicht tatenlos das nächste Jahrhunderthochwasser billigend in Kauf nehmen wollen, müssen wir endlich damit beginnen, Klimaschutz auch im Verkehr durchzusetzen. Dies verringert nicht nur die Folgen des Klimawandels, sondern spart auch eine Menge Geld.

AUTOR + KONTAKT

Michael Müller-Görnert ist Referent für Verkehrspolitik beim Verkehrsclub Deutschland (VCD)

Michael Müller-Görnert, Verkehrsclub Deutschland e.V. (VCD) Bundesverband, Rudi-Dutschke Straße 9, 10969 Berlin. Tel.: +49 30 280351-19,

E-Mail:michael.mueller-goernert@vcd.org, Internet: http://www.vcd.org 
Copyright (C) 2013, IÖW und oekom Verlag. Die Nutzung des Artikels ist Abonnenten von Ökologisches Wirtschaften vorbehalten. Nachdruck und Vervielfältigung des Artikels einschließlich Speicherung und Nutzung auf optischen und elektronischen Datenträgern nur mit Zustimmung der Redaktion von Ökologisches Wirtschaften (http://www.oekologischeswirtschaften.de). 